\title{
2. Die Klinik für Naturheilkunde und Integrative Medizin an den Kliniken Essen-Mitte, akademisches Lehrkrankenhaus der Universität Duisburg-Essen
}

Gustav Dobos

Die Klinik für Naturheilkunde und Integrative Medizin der Kliniken Essen-Mitte (ehemals. Innere Medizin V), Institutskennzeichen 260510 508, ist eine Krankenhausabteilung mit akutstationärem Versorgungsauftrag entsprechend dem Krankenhausplan des Landes NRW. Sie wurde 1999 an den Kliniken Essen-Mitte als Modelleinrichtung des Landes Nordrhein-Westfalen etabliert. Ziel ist es, optimale Behandlungsansätze aus konventioneller Medizin und wissenschaftlich evaluierter Naturheilkunde zu kombinieren. Neben dem breiten Spektrum der naturheilkundlichen Behandlungen bietet die Abteilung die kompletten diagnostischen und therapeutischen Möglichkeiten eines modernen Krankenhauses.

Angegliedert ist die Klinik den Kliniken Essen-Mitte, bestehend aus der Evang. Huyssens-Stiftung und dem Knappschafts-Krankenhaus. Dies sind Krankenhäuser der Schwerpunktversorgung mit den Fachabteilungen Innere Medizin, Onkologie, Senologie, Gynäkologische Onkologie, Chirurgie, Mund-, Kiefer- und Cesichtschirurgie, Urologie, Psychiatrie, Radiologie, Anästhesie, Pneumologie, Geriatrie und Naturheilkunde und verfügen insgesamt über 696 Planbetten mit ca. 1.200 beschäftigten Mitarbeitern.

Seit Oktober 2004 ist der Leiter der Klinik für Naturheilkunde und Integrative Medizin Inhaber des Stiftungslehrstuhls der Alfried Krupp von Bohlen und Halbach-Stiftung für Naturheilkunde mit den Schwerpunkten der Integration von Naturheilkunde in die konventionell bewährte Medizin und deren wissenschaftlicher Erforschung an der Universität Duisburg-Essen. Als Lehrkrankenhäuser 
tragen die Kliniken Essen-Mitte mit der Abteilung Naturheilkunde und Integrative Medizin zur Ausbildung im Bereich der Inneren Medizin und dem PJWahlfach Naturheilkunde bei. Die Abteilung verfügt zusammen mit der Abteilung für Gastroenterologie der Kliniken Essen-Mitte über die komplette internistische Weiterbildung von 5 Jahren.

Im Krankenhauspark wurde ein moderner Gebäudekomplex errichtet, in dem insgesamt 54 stationäre Betten, davon 12 integrativ-gastroenterologische Betten, zur Verfügung stehen. Die freundliche Atmosphäre der Abteilung bietet optimale Bedingungen zur Behandlung von Patienten mit chronischen Erkrankungen.

Angeschlossen an die Klinik sind die privatärztliche Ambulanz für Naturheilkunde und Traditionelle Chinesische Medizin, die Ambulanz für Traditionelle Indische Medizin sowie die privatärztliche Ambulanz für Integrative Gastroenterologie.

Weiterhin besteht zum einen ein umfangreiches tagesklinisches Angebot im Anschluss an einen stationären Aufenthalt. Zum anderen gibt es eine Tagesklinik mit onkologischem Schwerpunkt, die jedoch allen onkologischen Patienten offen steht. Die Verbindung aus ambulanter, stationärer und tagesklinischer Versorgung ist in dieser Form eine Novität in Deutschland und bietet die Möglichkeit einer individuellen Patientenbetreuung.

\subsection{Indikationen}

Indikationen bestehen für internistische Erkrankungen, insbesondere

- rheumatische Erkrankungen und rheumatischer Formenkreis

- chronische Schmerzerkrankungen (einschließlich Migräne)

- Asthma bronchiale, COPD

- funktionelle und chronisch entzündliche Darmerkrankungen

- Herz-Kreislauf-Erkrankungen (z.B. Bluthochdruck, KHK, pAVK)

- Metabolisches Syndrom

- onkologische Erkrankungen (adjuvant).

Am Standort Huyssens-Stiftung, der Kliniken Essen-Mitte, werden die Patienten der Abteilungen für Senologie (Brusterkrankungen), Gynäkologische Onkologie und Onkologie im Rahmen der Integrativen Onkologie neben der konventionellen Krebstherapie zusätzlich naturheilkundlich und mind-body-medizinisch unterstützend mitbehandelt.

\subsection{Diagnostische Verfahren}

Neben einer ausführlichen Anamnese und körperlichen Untersuchung unter Berücksichtigung spezieller naturheilkundlicher Aspekte wie manueller und 
reflektorischer Befunde, stehen sämtliche Untersuchungsoptionen der modernen Medizin (wie z.B.: Labordiagnostik, Stuhldiagnostik, Sonographie, Gastro- und Koloskopie, EKG, Langzeit-EKG und -Blutdruckmessung, ECHO, Lungenfunktionsprüfung, Röntgen, CT, MRT u.a.) zur Verfügung. Diese werden bei Bedarf in unserer Abteilung oder konsiliarisch durchgeführt.

\subsection{Therapeutische Verfahren}

Als sinnvolle Ergänzung der konventionellen Medizin kommen wissenschaftlich geprüfte Verfahren aus der klassischen und modernen Naturheilkunde, der Traditionellen Chinesischen Medizin sowie der Komplementärmedizin zum Einsatz.

Die Behandlung unserer Patienten erfolgt in der Regel durch ein interdisziplinäres Team, bestehend aus Ärzten, Pflegepersonal, Physiotherapeuten sowie Mitarbeitern der Ordnungstherapie. Wir bieten folgende Verfahren an:

- Ordnungstherapie/Mind-Body-Medizin (Stressbewältigung, Entspannungsverfahren, Yoga, QiGong, u.a.)

- Hydrotherapie (Güsse, Wassertreten, Wickel, Auflagen, Ölbäder nach Junge, Arm- und Fußbäder, Stangerbad, Zellenbäder, Elektrotherapie)

- Pflanzenheilkunde

- Bewegungstherapie (Bewegungsbad, Ergometertraining, Walking, Atmung und Aufrichtung)

- Ernährungstherapie und therapeutisches Fasten („Heilfasten“)

- Ausleitende Verfahren (Blutegel, Schröpfen, Baunscheidt, Kantharidenpflaster, Aderlass)

- Wärmeanwendungen (Hypertherapie, Wärmeauflagen, Wickel)

- Krankengymnastik und Physiotherapie (insbesondere Reflektorische Atemtherapie, Kraniosakrale- und Manuelle Therapie, Osteopathie, Fußreflexzonenmassage, CMD-Therapie sowie Feldenkrais)

- Akupunktur, NADA-Protokoll, Aku Taping, GuaSha,

- Neuraltherapie nach Huneke

- mikrobiologische Therapie und Symbioselenkung

In der Abteilung arbeiten Ärzte, die überwiegend langjährige Erfahrung in Innerer Medizin oder Allgemeinmedizin haben. Es bestehen Zusatzqualifikationen in Naturheilkunde, Physikalischer Therapie, Homöopathie, Akupunktur, Ernährungsmedizin, manueller Medizin und Chirotherapie, Neuraltherapie und Ayurveda-Medizin. Der Bereich der Ordnungstherapie/Mind-Body-Medizin wird durch diplomierte Psychologen, Gesundheits- und Sportpädagogen, Ökotrophologen sowie einen Iyengar-Yogalehrer vertreten. Im Team haben zudem pflegerisches Personal und ein spezialisiertes Team der Physiotherapie besondere Qualifikationen und Erfahrung. 
Ein individuelles Therapieprogramm wird für jeden Patienten in interdisziplinären Teamsitzungen zusammengestellt und im Verlauf des stationären Aufenthaltes den Bedürfnissen des Patienten, falls erforderlich, angepasst. Neben der Behandlung richtet sich der Fokus insbesondere auf das Vermitteln von Selbsthilfestrategien und die Förderung der Selbstkompetenz der Patienten. Positive Erfahrungen, die während des stationären Aufenthaltes im Sinne einer Beschwerdereduktion und einer Verbesserung des Allgemeinbefindens gemacht werden, wirken sich positiv auf die Compliance aus und erhöhen die Selbstwirksamkeit und damit die Wahrscheinlichkeit für einen anhaltenden Therapieerfolg über den stationären Aufenthalt hinaus. Unterstützt wird dieser Effekt durch ein Tagesklinikprogramm, dass im Anschluss des stationären Aufenthaltes von den Patienten wahrgenommen werden kann.

\subsection{Tagesklinik}

In der Tagesklinik finden über 1o Wochen einmal wöchentlich ordnungstherapeutisch geleitete Gruppen-Programme statt. Es wird eine Kombination aus Entspannungsverfahren und Stressbewältigung, gesunder Ernährung und Bewegung mit dem Ziel einer dauerhaften Lebensstilveränderung vermittelt und geübt. Durch zusätzliche ärztliche Visiten wird eine Beschwerdelinderung durch naturheilkundliche Selbsthilfestrategien gefördert. Ziel ist es, diese nachhaltig im Sinne einer anhaltenden Lebensstilmodifikation in den Alltag zu integrieren. Ebenso angegliedert ist eine Tagesklinik für onkologisch erkrankte Patienten, die an einem modifizierten Programm über 11 Wochen, häufig begleitend zur laufenden konventionellen Therapie, teilnehmen können.

\subsection{Ambulanzen}

Das Institut für Naturheilkunde, Traditionelle Chinesische und Indische Medizin bietet Möglichkeiten der Kombination konventioneller und naturheilkundlicher Behandlungen. Ein erfahrenes Team aus Ärzten und Heilpraktikern berät und behandelt mit klassischen naturheilkundlichen Verfahren sowie mit Therapien aus dem Bereich der Traditionellen Chinesischen Medizin (z.B. Zungen- und Pulsdiagnostik, Kräutertherapie, Akupunktur) und Indischen Medizin (Ayurveda). In der integrativen gastroenterologischen Ambulanz erhalten die Patienten eine ganzheitliche Behandlung, die konventionelle und naturheilkundliche Medizin sowie eine Lebensstilberatung umfasst. 


\subsection{Kostenübernahme}

Die gesetzlichen Krankenkassen übernehmen die Kosten für einen stationären Aufenthalt und die Tagesklinik. Bei einer privaten- oder Zusatzversicherung sollte die Kostenübernahme durch die Krankenversicherung im Vorfeld geklärt werden. Die Behandlungskosten werden über die Krankenhaustagessätze (DRG) bzw. die Gebührenordnung für Ärzte (GOÄ) abgerechnet. Die Abrechnung im ambulanten Bereich erfolgt privatärztlich.

\subsection{Patientennachfrage}

Es besteht eine hohe Nachfrage, für die ambulanten, stationären und teilstationären Behandlungen, sodass es zu Wartezeiten kommen kann. In der Regel erfolgt die Einweisung durch den behandelnden Haus- bzw. Facharzt.

\subsection{Wissenschaft und Forschung}

Ziel ist die Umsetzung erfahrungsorientierter Naturheilkunde in evidenzbasierte Medizin auf der Grundlage wissenschaftlicher Überprüfung. Bereits im Gründungsjahr 1999 wurden die ersten wissenschaftlichen Studien initiiert. 2004 wurde der Lehrstuhl für Naturheilkunde und eine Forschungsabteilung etabliert, 2007 folgte eine Studienambulanz für naturheilkundliche Verfahren und Phytotherapie. Seither wurden zahlreiche Publikationen in internationalen Peerreview Journals veröffentlicht, 2 Mitarbeiter habilitiert und etwa 35 Doktoranten promoviert.

Die wissenschaftliche Arbeit fokussiert sich hierbei auf folgende Bereiche:

- Hydrotherapie

- Neuraltherapie

- Phytotherapie

- Ausleitende Verfahren

- Ordnungstherapie/Mind-Body-Medizin

- Salutogenese/Lifestyle Medizin

- Traditionelle Chinesische Medizin (TCM)

- Traditionelle Ayurvedische Medizin

Eine Arbeitsgruppe, die aus Mitgliedern des Forschungsteams gegründet wurde, beschäftigt sich mit Methodenforschung und Leitlinienarbeit.

Durch den universitären Hintergrund und die damit verbundene enge Koppelung der Stationen und der Ambulanzen mit Forschung und Lehre ist eine zeitnahe Umsetzung neuer wissenschaftlicher Erkenntnisse im Bereich der Naturheilkunde gewährleistet. Dieses Wissen fließt in die studentische Ausbildung 
und der am Lehrstuhl angebotenen Fortbildungen für Ärzte zur Erlangung der Zusatzbezeichnung Naturheilverfahren oder auch in die Mind-Body-Medizin Fortbildung im Rahmen der Weiterbildungsakademie ein. 